\title{
Femtosecond dynamics of thin films by six-wave mixing
}

\author{
Shujie Lin, David L. Andrews, Ian D. Hands, Stephen R. Meech * \\ School of Chemical Sciences, University of East Anglia, Norwich NR4 7TJ, UK
}

Received 12 December 1997; in final form 20 January 1998

\begin{abstract}
The ultrafast dynamics of $10-\mu \mathrm{m}$-thick solutions of 4-dimethylamino- 4 ' nitrostilbene dye are measured by time-resolved non-degenerate six-wave mixing. The six-wave mixing origin of the optically induced second harmonic signal is confirmed. The time domain results are analysed in terms of a higher-order grating scattering model. The slowest relaxation time is shown to arise from orientational relaxation, through its viscosity dependence. It is shown that the observed orientational dynamics reveal the first and third moments of the molecular distribution function. These moments are not available from other experiments. It is also found that orientational relaxation cannot account for all the relaxation kinetics observed in polar solvents. It is proposed that the early time dynamics reflect a time-dependent change in the solute hyperpolarisability in polar solvents. (C) 1998 Elsevier Science B.V.
\end{abstract}

\section{Introduction}

In this Letter we report a femtosecond time domain six-wave mixing (SWM) study of the ultrafast solute dynamics in thin liquid films. The dynamics are probed by time-resolved second harmonic generation (SHG). The use of SHG as a probe of interface dynamics is established [1-4] but its application to solutions has been limited because of the well-known restriction that the conventional second-order SHG mechanism is (except in special circumstances [5,6]) rigorously forbidden in isotropic fluid media [7]. It has, however, been shown that SHG is allowed in isotropic media when higher-order multi-wave interactions are considered [8-10]. In the following it will be shown that SHG through ultrafast non-degenerate six-wave mixing provides novel information on the dynamics of thin films.

Higher-order time domain non-linear optical experiments have recently attracted a great deal of interest [11-13]. Several recent experiments have focused on non-resonant studies of the dynamics of pure liquids [14-18]. The two-dimensional nature of the six-wave mixing experiment provides in these cases a wealth of dynamical information which is not available through lower-order techniques [11-18]. There have also been some examples of electronically resonant six-wave mixing experiments, of which the earliest is perhaps light-induced SHG from isotropic solutions of a dye [19], also the subject of this work. The effect was ascribed to anisotropic bleaching of the ground state when the sample was irradiated with pulses at $\omega$ and $2 \omega$, resulting

\footnotetext{
* Corresponding author.
} 
in a medium with a finite $\chi^{(2)}$. The six-wave origin of the signal was demonstrated through its intensity and polarisation dependence [19]. Several potential applications of this anisotropic bleaching (optical poling) effect have been described [20]. Fiorini et al. also reported time-delayed SHG experiments with 30 ps time resolution, ascribing the observed relaxation observed to orientational relaxation [21]. More recently Fleming and co-workers reported a degenerate three-pulse six-wave mixing experiment with $50 \mathrm{fs}$ time resolution [22,23]. They showed that their experiment, like the non-resonant case, contains two-dimensional information through the multiple time delays. The (non-degenerate) six-wave mixing experiment described below has some similarities with that of Fleming and co-workers, but we will focus here on single time-delay (one-dimensional) measurements. In this case the six-wave mixing experiment may be represented as a higher-order form of transient grating scattering [19,21].

In Section 2 the experimental geometry will outlined. In Section 3 the sub-picosecond transient SWM measurements will be presented for a $10-\mu \mathrm{m}$-thick $10^{-3} \mathrm{M}$ solution of 4-dimethylamino-4'-nitrostilbene (DMANS). The data are described in terms of a higher-order grating scattering model. In Section 4 the transient data recorded in three solvents of different polarity and viscosity are presented. These are analysed in terms of solute reorientational relaxation and excited state evolution. The conclusions are summarised in the final section.

\section{Experimental}

The source used in the experiments described below was a regeneratively amplified titanium sapphire laser operating at $800 \mathrm{~nm}$. The output pulsewidth from the compressor was measured by background-free autocorrelation to be $70 \mathrm{fs}$. The repetition frequency was $5 \mathrm{kHz}$. The optical geometry for the six-wave mixing experiment, shown in Fig. 1, was based on a phase conjugate arrangement [19,21]. Vertically polarised $800 \mathrm{~nm}$ pulses were first sent through a telescope to reduce the beam size and then divided at beamsplitter, BS1 (reflectivity 50\%). The transmitted beam (beam 1) was routed through a fixed optical path to the sample. The reflected beam was further divided at a second $50 \%$ reflecting beamsplitter, BS2. The reflected part (beam 2) was routed through a translatable delay stage $\left(t_{12}\right.$, accuracy $\left.2 \mu \mathrm{m}\right)$, its polarisation rotated $90^{\circ}$ by a half-wave plate before entering a $300-\mu \mathrm{m}$-thick BBO crystal for type I second harmonic generation. The vertically polarised second harmonic output from the crystal was passed through a bandpass filter to remove residual $800 \mathrm{~nm}$ light and then directed onto the sample via a 50\% reflecting beamsplitter, BS3. Beams 1 and 2, with an angle of $4.5^{\circ}$ between them,

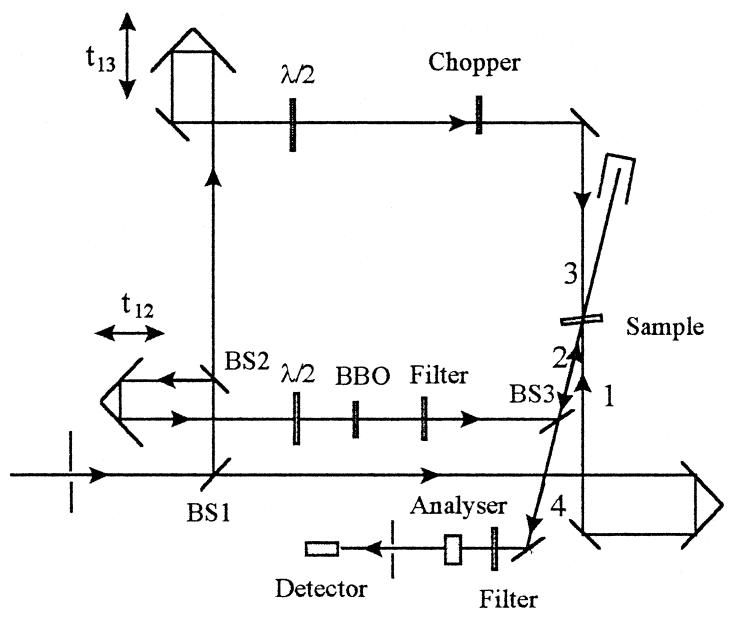

Fig. 1. Optical arrangement for the femtosecond phase conjugate six-wave mixing measurement. For a detailed description see text. 
were spatially and temporally overlapped by the observation of sum frequency generation $(\omega+2 \omega)$ in a second BBO crystal located at the sample position. Using this arrangement and varying $t_{12}$ the pulsewidth at the sample was measured as $100 \mathrm{fs}$. The beam transmitted by BS2 (beam 3) was routed through a motorised delay stage $\left(t_{13}\right.$, positional accuracy $\left.0.1 \mu \mathrm{m}\right)$, a half-wave plate and then onto the sample in a direction exactly counterpropagating with respect to beam 1 . With this geometry the second harmonic signal (beam 4 ) generated in the sample was emitted back along the path of beam 2. This signal was transmitted by BS3, passed through a bandpass filter, an analysing polariser and a spatial filter before detection by a monochromator photomultiplier tube combination. The output of the photomultiplier was processed by a lock-in amplifier referenced to the frequency of a chopper placed in the path of beam 3. Both $t_{13}$ and the lock-in were under computer control.

The samples were $10 \mu \mathrm{m}$ pathlength solutions of DMANS contained between two silica plates separated by a layer of foil. All solvents were of spectroscopic grade. The absorption maximum was at approximately $420 \mathrm{~nm}$, and weakly dependent on solvent. The solute concentration was $2 \times 10^{-3} \mathrm{M}$ yielding an optical density of 0.2 . The three beams were weakly focused into the sample by the telescope, yielding a beam diameter of approximately $800 \mu \mathrm{m}$. The final peak powers were $80 \mathrm{GW} \mathrm{cm}^{-2}$ (beam 1), $1.0 \mathrm{GW} \mathrm{cm}^{-2}$ (beam 2), and 40 GW $\mathrm{cm}^{-2}$ (beam 3). It was established by absorption spectroscopy that sample degradation was negligible during the measurements.

\section{Six-wave mixing measurements}

The SHG signal detected in the geometry of Fig. 1 was measured as a function of the delay $t_{13}$ with $t_{12}$ set equal to zero. The result for a solution of DMANS in THF is shown in Fig. 2 as the signal amplitude, $E_{4}(2 \omega) \propto I^{1 / 2}(2 \omega)$. Fig. 2 shows a symmetrical spike around $t_{13}=0$, a rapid risetime $(0.7 \pm 0.2 \mathrm{ps})$ followed by a biexponential decay with time constants of 1.9 and 86 ps. The six-wave origin of this signal was confirmed from its quadratic dependence of the signal intensity on the intensity of beams 1 and 3, and a linear dependence on the intensity of beam 2 . These data should be compared with the one previous time-resolved study of induced SHG in solution [21] where only a time zero spike and an exponential decay were reported. Evidently the sub-picosecond measurement reveals some novel dynamics.

For the particular case considered here, $t_{12}=0$ with a time-delayed probe at $t_{13}$, it is useful to discuss the

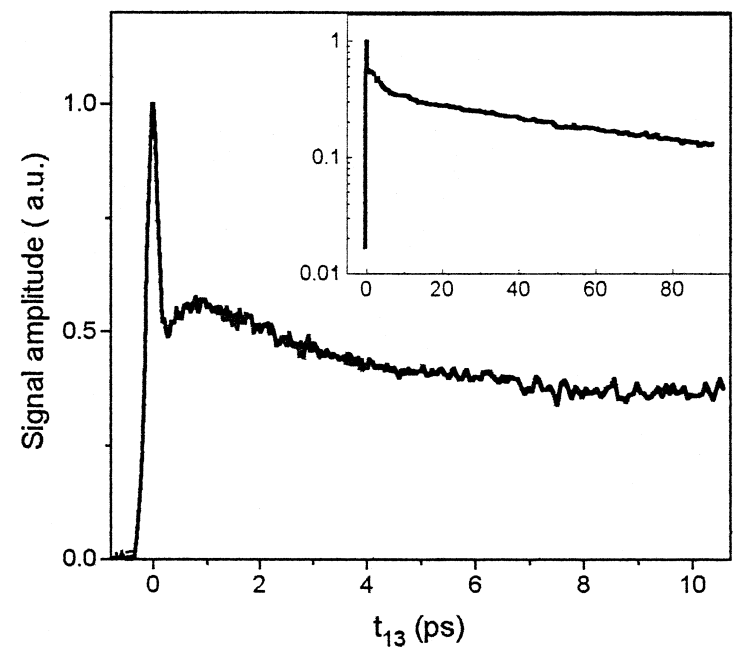

Fig. 2. Amplitude of the six-wave mixing signal from the $10-\mu \mathrm{m}$-thick $10^{-3} \mathrm{M}$ DMANS solution in THF. Data are shown as a function of the delay $t_{13}$ between pulse 3 and the pulses 1 and 2, which are timed to arrive simultaneously at the sample $\left(t_{12}=0\right)$. All fields are vertically polarised. Note the sub-picosecond risetime and biexponential decay; the latter is most clearly seen in the logarithmic plot (inset) of the long time data. 
time-resolved SWM data in terms of a higher-order grating scattering experiment. In this case pulses 1 (wavevector $\left.\vec{k}_{1}\right)$ and $2\left(\vec{k}_{2}\right)$ interact in the sample to write a spatial grating in the ground state population of the form [21]

$$
\begin{aligned}
P_{10}(\theta, \phi, \vec{r}) & =\frac{\mu^{2} \Delta \mu E_{1}^{2}(\omega) E_{2}(2 \omega) \tau^{2}}{4 \hbar^{3} \omega}\left[\hat{\mu} \cdot \hat{e}_{1}(\omega)\right]^{2}\left[\hat{\mu} \cdot \hat{e}_{2}(2 \omega)\right] \cos \left[\left(\vec{k}_{2}(2 \omega)-2 \vec{k}_{1}(\omega)\right) \cdot \vec{r}+\varphi_{0}\right] \\
& =\rho(\theta, \phi, t=0) \cos \left[\left(\vec{k}_{2}(2 \omega)-2 \vec{k}_{1}(\omega)\right) \cdot \vec{r}+\varphi_{0}\right],
\end{aligned}
$$

where the $\theta$ and $\phi$ polar angle dependence of the scalar products on the right is implicit. In Eq. (1) $P_{10}$ is the spatially modulated part of the excitation probability arising from the interference of beams 1 and 2 , which is a function of both the position vector $\vec{r}$ and the orientation of the transition dipole $\boldsymbol{\mu}$ with respect to the laboratory axis. For the case of DMANS it may be assumed that both the permanent and transition dipole moments lie along the direction of the molecular axis. In general this approximation is not necessary (see [24]) but will be made here for simplicity. The other factors are the change in permanent dipole moment on excitation, $\Delta \mu$, the field amplitudes, $E_{\mathrm{i}}$, the unit polarisation vectors, $\tilde{A_{\mathrm{i}}}$, the pulse width, $\tau$, and the initial phase difference between pulses 1 and $2, \varphi_{0}$. The orientational part of Eq. (1), $\rho(\theta, \phi, t=0)$, determines the initial $(t=0)$ molecular orientational distribution function. Electronic excitation by pulses 1 and 2 individually also occurs, but the resultant probability distribution from these contributions exhibit inversion symmetry, and so cannot contribute to the SHG signal; they merely provide a constant, unmodulated, background excitation probability [24].

The grating description (Eq. (1)) has a number of important features. Firstly, it suggests how SHG can occur in isotropic solutions through the interference of beams 1 and 2 . It can be seen that $\left(\hat{\mu} \cdot \hat{e}_{2}(2 \omega)\right)$ changes sign when the molecule is inverted, while $\left(\hat{\mu} \cdot \hat{e}_{1}(\omega)^{2}\right)$ does not. As a result the contribution to the total excitation probability arising from Eq. (1) also changes sign and so generates within the sample a medium without inversion symmetry, so SHG is allowed; essentially a $\chi^{(2)}$ grating is formed. For the particular case of all beams vertically polarised, as in Fig. 2, the excitation probability is proportional to $\cos ^{3} \theta$, where $\theta$ is angle between the molecular and laboratory axis. Secondly, the $\vec{k}_{2}-2 \vec{k}_{1}$ grating vector of Eq. (1) predicts the observed signal direction, since when $\vec{k}_{3}=-\vec{k}_{1}$, phase matching occurs for the signal $\vec{k}_{4}=-\vec{k}_{2}$.

The above analysis in terms of a $\chi^{(2)}$ grating induced by beams 1 and 2 , and probed by beam 3 , is not adequate at very early times $\left(t_{12} \approx t_{13} \approx 0\right)$ associated with the so-called coherence spike. In that case, the distinction between pump and probe beams cannot be made, and a full SWM treatment is more appropriate and may indeed contain additional dynamical information [24]. However, with the current geometry this information is not available because of an intrinsic limitation on time resolution in the phase conjugate geometry. Essentially, the time resolution in this geometry is a convolution of both pulse width and sample optical length. The effect is well known from ultrafast phase conjugate four-wave mixing experiments [25]. It was observed that, if the pathlength in the solution was increased to $100 \mu \mathrm{m}$, both the coherence spike and the sub-picosecond risetime seen in Fig. 2 were obscured, and only the biexponential decay was observed.

In Section 4 the dynamics observed in Fig. 2 are analysed in terms of the induced $\chi^{(2)}$ grating model described above. The complete description of the SWM signal is to be presented elsewhere [24].

\section{Analysis and discussion}

The SHG signal generated from beam 3 at time $t_{13}$ reflects the decay time of the induced $\chi^{(2)}$ grating. This may occur through excited state relaxation and solute reorientation, which may be expressed as

$$
\begin{gathered}
E_{4}(2 \omega, t) \propto \int\left[\hat{\mu} \cdot \hat{e}_{4}(2 \omega)\right]\left[\hat{\mu} \cdot \hat{e}_{3}(\omega)\right]^{2} \Delta \beta(t) N_{1}(t) \rho(\theta, \phi, t) \sin \theta \mathrm{d} \theta \mathrm{d} \phi \\
=N_{1}(t) \Delta \beta(t) \int\left[\hat{\mu} \cdot \hat{e}_{4}(2 \omega)\right]\left[\hat{\mu} \cdot \hat{e}_{3}(\omega)\right]^{2} \rho(\theta, \phi, t) \sin \theta \mathrm{d} \theta \mathrm{d} \phi
\end{gathered}
$$


where $N_{1}(t)$ is the time-dependent population of the excited state and $\Delta \beta(t)$ is the change in molecular hyperpolarisability on excitation (which, for the rod-like DMANS molecule, can be assumed to have a significant non-zero component only along the molecular axis, $\hat{\mu}$ ). In general $\Delta \beta$ is time dependent. The time-dependent orientational distribution function, $\rho(\theta, \phi, t)$, has an initial $(t=0)$ distribution given by Eq. (1). The fluorescence quantum yield of DMANS in THF is high, suggesting that excited state population relaxation will not contribute significantly to the observed relaxation on the 100 ps timescale. On the other hand, the 100 ps timescale is precisely that expected for orientational relaxation of molecules of the size of DMANS in fluid solvents. Eq. (2) leads, via a solution of the diffusion equation by standard methods [21,27] to

$$
E_{4}(2 \omega, t) \propto N_{1}(t) \Delta \beta(t)\left[\frac{4}{7} \mathrm{e}^{-12 D t}+3 \mathrm{e}^{-2 D t}\right],
$$

for all vertical polarisation of beams $1-4$, where $D$ is the orientational diffusion coefficient, and the values 2 and 12 in the exponent arise from contributions from the first $(l=1)$ and third $(l=3)$ moments of the orientational distribution function [21,26,27]. In arriving at Eq. (3) a symmetric top model for the rod like DMANS molecule was again assumed. Assuming that the entire time dependence of $E_{4}(2 \omega, t)$ can be ascribed to orientational relaxation, Eq. (3) predicts that the signal will decay as a biexponential function, as was observed beyond about 1 ps (Fig. 2). However, the measured relaxation times in THF differ by a factor of 40, rather than 6 as predicted by Eq. (3). This suggests that factors other than orientational relaxation might contribute to the relaxation.

To further test the orientational relaxation model the measurement on THF was repeated with a different set of initial polarisation conditions, specifically beams 1 and 2 vertically polarised, beam 3 at $45^{\circ}$ and the analyser set to record the horizontal component of beam 4. With these new initial conditions Eq. (2) yields

$$
E_{4}^{\prime}(2 \omega, t) \propto N_{1}(t) \Delta \beta(t)\left[-\frac{2}{7} \mathrm{e}^{-12 D t}+\mathrm{e}^{-2 D t}\right] .
$$

Taking the ratio of Eq. (3) and (4) yields a result which is independent of any contribution to the relaxation from a time-dependent excited state population or hyperpolarisability,

$$
r(t)=\frac{4 \mathrm{e}^{-12 D t}+21 \mathrm{e}^{-2 D t}}{-2 \mathrm{e}^{-12 D t}+7 \mathrm{e}^{-2 D t}}=\frac{21+4 \mathrm{e}^{-10 D t}}{7-2 \mathrm{e}^{-10 D t}} .
$$

The results for the measurements corresponding to Eqs. (3), (4) and their ratio (5) are shown in Fig. 3. The time-dependent data described by Eqs. (3) and (4) reveal a noticeably different time dependence especially in the region 1-20 ps, which is where the $12 \mathrm{D}$ contribution to the relaxation would be expected to have an effect (assuming the long relaxation time corresponds to $2 D$ ). That both the first $(2 D)$ and third $(12 D)$ moments of the molecular orientational distribution function contribute to the observed relaxation dynamics is confirmed by the ratio (5), where the data are fit assuming $1 / 2 D=86 \mathrm{ps}$. The trace decays from the expected value of 5 at early time to 3 at times where the contribution of $12 \mathrm{D}$ is insignificant. This analysis demonstrates that the SWM measurement described is an experimental tool for the determination of the odd moments of the molecular orientation; these are not available from other measurements, such as fluorescence anisotropy and four-wave mixing [26,27]. Of course no new dynamical information is available from this measurement for the present case of the rod like DMANS molecule in an isotropic solution, where only a single diffusion coefficient is required to describe the orientational relaxation. However, it has recently been shown that the determination of different moments of the orientational distribution function is essential for the complete description of orientational relaxation in anisotropic media, such as liquid crystals [27].

A second conclusion from the above analysis is that orientational relaxation does not describe the complete time-delayed SWM signal, in particular the risetime and 1.9 ps relaxation seen in Fig. 2. This is further emphasised in Fig. 4a, where the SWM data measured in three solvents are presented. The data are compared with the result predicted if orientational relaxation is the only contribution to the relaxation (the value for $2 D$ was taken from the longest observed relaxation time, i.e for $t>40 \mathrm{ps}$ ). Clearly in the two polar solvents 


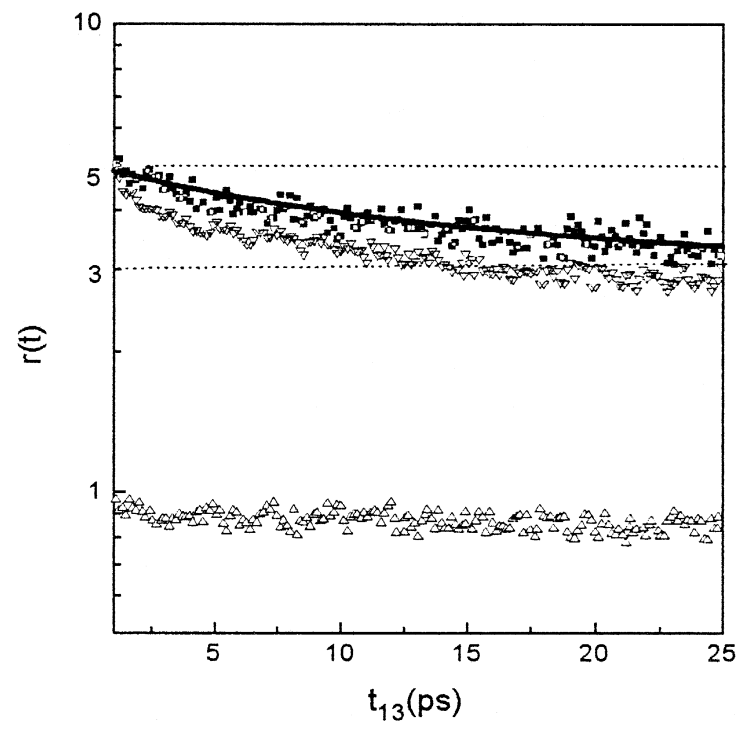

Fig. 3. The time dependence of the ratio $(\boldsymbol{\square})$ of the signal amplitudes with the two different polarisation arrangements: $(\nabla)$ is the measured data for all vertical polarisations (Eq. (3)) and $(\Delta)$ for vertical beams 1 and 2, beam 3 at $45^{\circ}$ and detection of the horizontal component of beam 4 (Eq. (4)). The ratio agrees well with the theoretical prediction of (Eq. (5)) (solid line).

orientational relaxation does not describe the first few picoseconds of the data at all well, while in the non-polar (toluene) case it accounts well for the entire relaxation. It may further be noted that the dependence on viscosity of the measured long relaxation time provides additional confirmation that orientational diffusion is its relaxation mechanism; the measured value of $1 / 2 \mathrm{D}$ was observed to be a linear function of solvent viscosity.

The non-diffusional part of the relaxation appears in Fig. $4 \mathrm{a}$ as a rapid risetime and 1.9 ps decay in THF, an unresolved risetime and 15 ps decay in anisole, and is essentially absent in toluene. Three explanations can be considered. Firstly, non-diffusive solute orientational relaxation - librational dynamics - could contribute to the early time response. This cannot, however, be the explanation for the data of Fig. 4a, since it is unlikely that the librational response would be absent in toluene, but present in THF and anisole, when toluene is of intermediate viscosity. The second possible influence on the dynamics is population relaxation (Eq. (2)). However, the quantum yield is high, hence excited state lifetime is long, in all solvents studied. This suggests that population relaxation is not the explanation for the fast relaxation kinetics observed.

A final possibility for the non-orientational contribution to the relaxation seen in Fig. 4a is a time-dependent value of $\Delta \beta$ (Eq. (2)). In complex molecules like DMANS the hyperpolarisability is represented by a sum over many states, and is a function of the electronic structure and energy of those states [28]. Theoretical calculations suggest that at least the ground and first three excited states contribute to the large non-linear optical coefficients of DMANS [29,30]. We suggest that the non-orientational contribution to the relaxation of the SWM signal arises from the effect on $\Delta \beta$ of an evolution of the energy separation (and hence resonance enhancement) and permanent dipole moment of these states following electronic excitation.

The non-orientational part of the SWM response is isolated by dividing the experimental data of Fig. 4a with the calculated orientational relaxation (Eq. (3)). The result is shown in Fig. 4b. The non-orientational relaxation is highly solvent dependent, being absent in toluene, showing a sub-picosecond rise and rapid decay in THF and a non-exponential relaxation in anisole; clearly this contribution to the relaxation is induced by polar solvents. In addition the timescale of the relaxation in Fig. $4 \mathrm{~b}$ scales with the dielectric relaxation time of the solvent. For THF the dielectric relaxation time is $3.1 \mathrm{ps}$ (obtained from a Cole-Cole dielectric dispersion [31]) while for anisole the main contribution of the observed double Debye type dispersion has a relaxation time of 14.7 ps 

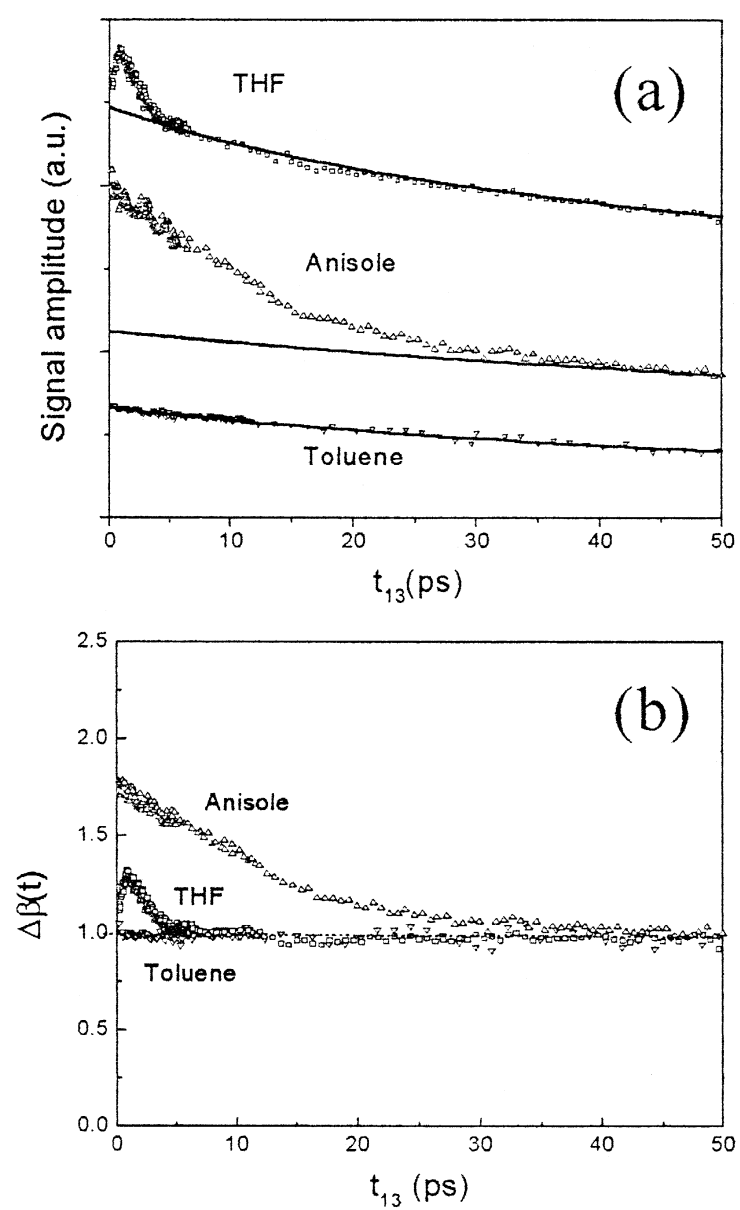

Fig. 4. (a) SWM signal amplitudes from the DMANS solutions in THF, anisole, and toluene as functions of the delay time with all vertical polarisations. The solid curves are the dynamics predicted by the orientational relaxation model. Note the curves for THF and anisole have been shifted for clarity. (b) The time dependence of the hyperpolarisabilty change $\Delta \beta$ of DMANS in THF, anisole and toluene. The rapid rise and decay in THF, a slower decay in anisole and no resolvable change in toluene are consistent with a dynamic solvent stabilisation of a ICT state in DMANS (see text).

[32]. From these results it is concluded that the time-dependent $\Delta \beta$ arises from the polar solvent stabilisation of the electronic states of DMANS.

The low-lying electronic states of DMANS have, in common with other donor-acceptor substituted stilbenes, a high degree of intramolecular charge transfer (ICT) character [33]. The degree of ICT can be enhanced by polar solvent stabilisation accompanied by nuclear reorganisation. For example in a related donor acceptor stilbene dye, 4-dimethylamino-4'-cyanostilbene, the large Stokes shift was assigned to the formation of an ICT state in polar solvents arising through twisting about the ethene-dimethylamino bond [33]. The dynamics of the stabilisation will reflect the reorganisation time of the solvent to accommodate the newly created ICT state. Solvent stabilisation of ICT states has been studied in great detail [34]. At least in simple cases the dynamics are controlled by the solvent reorientation time. The dynamics are expected [35] to show a component of $\approx 100 \mathrm{fs,}$ not resolvable from the time zero response in the current experiment, and a component with a relaxation time lying between the solvent's longitudinal relaxation and Debye relaxation times. The data of Fig. $4 \mathrm{~b}$ can then be 
understood as arising from the stabilisation by solvent reorientation of an ICT state with a different electronic structure, and hence a different $\Delta \beta$, compared to that initially formed on excitation.

\section{Conclusions}

The application of SHG from isotropic solutions as a probe of the ultrafast solute dynamics of thin films has been described. The SHG signal was shown to arise through a SWM mechanism. The observed time-resolved data were described in terms of a concerted SWM signal at $t=0$ and a consecutive mechanism when the beam generating the SHG signal was time delayed. The former contribution could not be time resolved in the current experiment. The latter was described in terms of a higher-order transient grating scattering mechanism.

Analysis of the time-resolved data revealed a highly non-exponential relaxation. This could be assigned to two mechanisms: solute reorientation and a solvent dependent evolution of the solute excited state. It has been shown that the analysis of the orientation relaxation reveals the first and third moments of the molecular distribution function, and these were resolved for the first time. These parameters are not accessible through lower-order optical experiments such as fluorescence and four-wave mixing.

The solute reorientation was found to account for the entire relaxation in toluene, but in polar solvents an additional relaxation component was observed. This was ascribed to the solvent stabilisation of an ICT state by solvent reorientation. Such an evolution of the electronic structure of the excited state will lead to a time-dependent value of $\Delta \beta$, and so be reflected in the time-resolved SWM signal.

\section{Acknowledgements}

We are grateful to EPSRC for support of this research.

\section{References}

[1] S.R. Meech, in: S.H. Lin, A. Villaeys, Y. Fujimura (Eds.), Advances in Multiphoton Processes and Spectroscopy (World Scientific, 1993) p. 281.

[2] E.V. Sitzmann, K.B. Eisenthal, J. Phys. Chem. 92 (1988) 4579.

[3] S.R. Meech, K. Yoshihara, Chem. Phys. Lett. 154 (1989) 20.

[4] Y.R. Shen, The Principles of Nonlinear Optics (John Wiley, 1984).

[5] D.L. Andrews, P. Allcock, A.A. Demidov, Chem. Phys. 190 (1995) 1.

[6] P. Allcock, D.L. Andrews, S.R. Meech, A. Wigman, Phys. Rev. A 53 (1996) 2788.

[7] D.L. Andrews, N.P. Blake, Phys. Rev. A 38 (1988) 3113.

[8] D.L. Andrews, Nonlinear Opt. 8 (1994) 25.

[9] P. Allcock, D.L. Andrews, J. Phys. B: Atom. Mol. Opt. Phys. 30 (1997) 3731.

[10] M. Ducloy, Appl. Phys. Lett 46 (1985) 1020.

[11] P.F. Barbara, J.G. Fujimoto, W.H. Knox, W. Zinth (Eds.), Ultrafast Phenomena X, Springer Series in Chemical Physics Vol. 62 (Springer, Berlin, 1996).

[12] Y. Tanimura, S. Mukamel, J. Chem. Phys. 99 (1993) 9496.

[13] S. Mukamel, Principles of Nonlinear Optics (OUP, New York, 1995).

[14] K. Tominaga, K. Yoshihara, J. Chem. Phys. 104 (1996) 1159.

[15] K. Tominaga, K. Yoshihara, J. Chem. Phys. 104 (1996) 4419.

[16] K. Tominaga, K. Yoshihara, Phys. Rev. Lett. 74 (1995) 3061.

[17] T. Steffen, K. Duppen, Phys. Rev. Lett. 76 (1996) 1224.

[18] T. Steffen, K. Duppen, J. Chem. Phys. 106 (1997) 3854.

[19] F. Charra, F. Devaux, J.-M. Nunzi, P. Raimond, Phys. Rev. Lett. 68 (1992) 2440.

[20] C. Fiorini, J.-M. Nunzi, F. Charra, F. Kajzar, M. Lequan, R.-M Lequan, K. Chane-Ching, Chem. Phys. Lett. 335 (1997) 271. 
[21] C. Fiorini, F. Charra, J.-M. Nunzi, J. Opt. Soc. Am. B 11 (1994) 2347.

[22] M. Cho, G.R. Fleming, J. Phys. Chem. 98 (1994) 3478.

[23] T. Joo, Y. Jia, G.R. Fleming, J. Chem. Phys. 102 (1995) 4063.

[24] I.D. Hands, S. Lin, S.R. Meech, D.L. Andrews, manuscript in preparation.

[25] M. Zhao, Y. Cui, M. Samoc, P.N. Prasad, M.R. Unroe, B.A. Reinhardt, J. Chem. Phys. 95 (1991) 3991.

[26] A.J. Bain, P. Chandna, G. Butcher, Chem. Phys. Lett. 260 (1996) 441.

[27] A.J. Bain, P. Chandna, J. Bryant, manuscript submitted.

[28] J.F. Ward, Rev. Mod. Phys. 37 (1965) 1.

[29] D. Beljonne, J.L. Bredas, M. Cha, W.E. Torruellas, G.I. Stegeman, G.W. Hofstraat, W.H.G. Horsthuis, G.R. Mohlmann, J. Chem. Phys. 103 (1995) 7834.

[30] D. Beljonne, J.L. Bredas, G. Chen, S. Mukamel, Chem. Phys. 210 (1996) 353.

[31] S.K. Garg, C.P. Smyth, J. Chem. Phys. 42 (1965) 1397.

[32] S.K. Garg, C.P. Smyth, J. Chem. Phys. 46 (1967) 373.

[33] W. Rettig, W. Majenz, Chem. Phys. Lett. 154 (1989) 335.

[34] M. Maroncelli, J. Mol. Liq. 57 (1993) 1.

[35] M.L. Horng, J.A. Gardecki, M. Maroncelli, J. Phys. Chem. 99 (1995) 17311. 